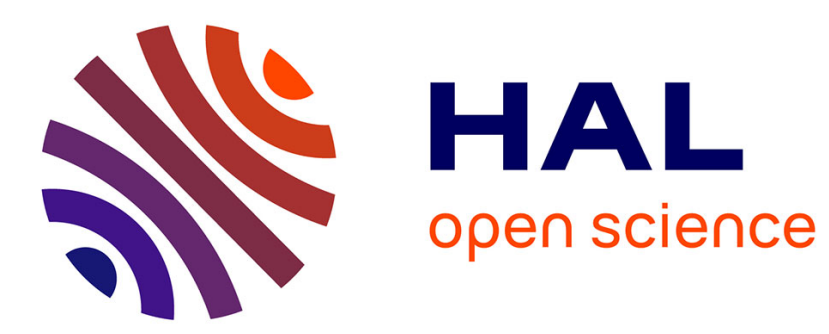

\title{
Nonlinear thresholding of multiresolution decompositions adapted to the presence of discontinuities
}

Sergio Amat, Jacques Liandrat

\section{- To cite this version:}

Sergio Amat, Jacques Liandrat. Nonlinear thresholding of multiresolution decompositions adapted to the presence of discontinuities. Advances in Computational Mathematics, 2013. hal-01266099

\section{HAL Id: hal-01266099 \\ https://hal.science/hal-01266099}

Submitted on 3 Feb 2016

HAL is a multi-disciplinary open access archive for the deposit and dissemination of scientific research documents, whether they are published or not. The documents may come from teaching and research institutions in France or abroad, or from public or private research centers.
L'archive ouverte pluridisciplinaire HAL, est destinée au dépôt et à la diffusion de documents scientifiques de niveau recherche, publiés ou non, émanant des établissements d'enseignement et de recherche français ou étrangers, des laboratoires publics ou privés. 


\title{
Nonlinear thresholding of multiresolution decompositions adapted to the presence of discontinuities
}

\author{
Sergio Amat* Jacques Liandrat ${ }^{\dagger}$
}

July 22,2011

\begin{abstract}
A new nonlinear representation of multiresolution decompositions and new thresholding adapted to the presence of discontinuities are presented and analyzed. They are based on a nonlinear modification of the multiresolution details coming from an initial (linear or nonlinear) scheme and on a data dependent thresholding. Stability results are derived. Numerical advantages are demonstrated on various numerical experiments.
\end{abstract}

Key Words. Multiresolution, nonlinear thresholding, stability, compression.

AMS(MOS) subject classifications. 41A05, 41A10, 65D05, 65D17

\section{Introduction}

This paper is devoted to the analysis of a new nonlinear multiresolution representation scheme and associated thresholding for discrete data. Given $f^{L}$ a data where $L$ stands for a resolution level, a multiresolution representation of $f^{L}$ is any sequence of type $\left\{f^{0}, d^{0}, \ldots, d^{L-1}\right\}$ where $f^{j}$ is an approximation of $f^{L}$ at resolution $j<L$ and $d^{j}$ stands for the details required to recover

${ }^{*}$ Departamento de Matemática Aplicada y Estadstica. Universidad Politécnica de Cartagena (Spain). Research supported in part by the Spanish grants MICINN-FEDER MTM2010-17508 and 08662/PI/08. e-mail:sergio.amat@upct.es

${ }^{\dagger}$ Centrale Marseille, LATP, 13451 Marseille, France email:jacques.liandrat@centrale-marseille.fr 
$f^{j+1}$ from $f^{j}$. The couple $\left\{f^{j}, d^{j}\right\}$ contains the same information as $f^{j+1}$ and therefore the same is true for $\left\{f^{0}, d^{0}, \ldots, d^{L-1}\right\}$ and $f^{L}$.

Inter-resolution operators are named decimation (from fine, i.e. large value of $j$, to coarse, i.e. small value of $j$ ) and prediction (from coarse to fine). The reconstruction and discretization operators act between the continuous level and any discrete level (see [6], [15] for more details).

Linear multiresolution representations of data are multiresolutions involving linear inter-resolution operators. It turns out that the efficiency of linear multiresolution decompositions, for instance for image compression, is generally limited by the presence of discontinuities or edges, since the detail coefficients $d^{j}$ close to the discontinuities remain significant even when $j \rightarrow+\infty$. In wavelet multiresolution for instance, the numerically significant detail coefficients $d_{k}^{j}$ are mainly those for which the corresponding wavelet support is intersected by discontinuities.

Different steps towards adaption near singularities has been proposed involving nonlinear prediction operators [2], [3], [5], [6], [7], [8], [9], [14], [16], [18].

The aim of this paper is to describe and analyze a new nonlinear representation for multiresolution transforms and new thresholding strategy that incorporate the presence of discontinuities. It is based on a nonlinear modification of the multiresolution details generated by an initial scheme and on a data dependent thresholding. We present several results related to the stability of these multiresolution schemes. Numerical properties are demonstrated on various experiments.

Our strategy is related to the notion of normal approximation for curves or surfaces that can be found in [9], [13]. A multiresolution approximation of a curve or surface is normal if all the detail vectors align with a locally defined normal direction which only depends on the coarser levels. Because they depend on the computation of a normal, these approximations lead to nonlinear representations. When one uses normal multiresolution only a single scalar coefficient needs to be stored instead of the standard 2-D or 3 -D vector; memory saving is then performed. It turns out to be that the direction of the normal provides also some information on the signal, that can be used for better compression in the presence of discontinuities.

The paper is organized as follows: we recall in section 2 the discrete pointvalue framework for multiresolution and its relation with subdivision schemes. Our algorithm is then described and analyzed theoretically in sections 3, 4 and 7. Numerical performances are presented in section 8 and finally some conclusions are provided in section 9 . 


\section{The interpolatory multiresolution setting}

Let us consider a set of nested grids in $[0,1]$ :

$$
X^{j}=\left\{x_{k}^{j}\right\}_{k=0}^{J_{j}}, \quad x_{k}^{j}=k h_{j}, \quad h_{j}=2^{-j} / J_{0}, \quad J_{j}=2^{j} J_{0},
$$

where $J_{0}$ is some fixed integer and the point-value discretization operator

$$
\mathcal{D}_{j}: \begin{cases}C([0,1]) & \rightarrow V^{j} \\ f & \mapsto f^{j}=\left(f_{k}^{j}\right)_{k=0}^{J_{j}}=\left(f\left(x_{k}^{j}\right)\right)_{k=0}^{J_{j}}\end{cases}
$$

where $V^{j}$ is the space of real sequences of length $J_{j}+1$ and $C([0,1])$ the set of continuous functions on $[0,1]$. A reconstruction operator $\mathcal{R}_{j}$ associated to this discretization is any right inverse of $\mathcal{D}_{j}$ on $V_{j}$ which means that

$$
\left(\mathcal{R}_{j} f^{j}\right)\left(x_{k}^{j}\right)=f_{k}^{j}=f\left(x_{k}^{j}\right) .
$$

The operator $\mathcal{D}_{j+1} \mathcal{R}_{j}$ provides a subdivision scheme. Moreover, since $\mathcal{D}_{j+1} \mathcal{R}_{j} f^{j} \neq f^{j+1}$ for any function $f$, details should be added to recover $f^{j+1}$ from $f^{j}$ and multiresolution transforms are then available (see [6] for details).

\subsection{Data independent Lagrange interpolation}

Given two integers $r \geq 1, r \geq s>0$, data independent Lagrange interpolation can be defined using a fixed set of indices (a stencil)

$$
\mathcal{S}=\mathcal{S}(r, s)=\{-s,-s+1, \ldots,-s+r\}, \quad r \geq s>0, r \geq 1,
$$

such that the predicted value at scale $j+1$ and position $x_{2 k+1}^{j+1}$ is the value at the same position of $\mathcal{I}_{j}\left(x, f^{j}\right)$, the Lagrange polynomial of degree $r$ interpolating the set of values $\left\{f_{k+m}^{j}, m \in \mathcal{S}\right\}$ at positions $\left\{x_{k+m}^{j}, m \in \mathcal{S}\right\}$.

Linearity and reproduction of polynomial of degree less or equal to $r$ implies that for any function $f \in C^{r+1}$,

$$
f_{j}=f\left(x_{k}^{j}\right) \Rightarrow \mathcal{I}_{k}\left(x_{2 k+1}^{j+1}, f^{j}\right)=f\left(x_{2 k+1}^{j+1}\right)+O\left(h_{j}\right)^{r+1} .
$$

The prediction procedure for smooth data is then said to be of accuracy $p=r+1$.

The Lagrange interpolatory techniques lose much of their accuracy in the presence of isolated singularities. Indeed, if there exists a discontinuity 
point of $f$ in $\left[x_{k-1}^{j}, x_{k}^{j}\right]$, it is easy to check that any divided difference ${ }^{1}$ based on a set of $s+1$ points containing $\left\{x_{k-1}^{j}, x_{k}^{j}\right\}$ verifies

$$
f\left[x_{l}^{j}, \ldots, x_{l+s}^{j}\right]=O\left(\Delta_{k}^{j}\right) / h_{j}^{s},
$$

where $\Delta_{k}^{j}=f_{k}^{j}-f_{k-1}^{j}$. Therefore, for any polynomial piece $\mathcal{I}_{l}(x)$ which stencil $\mathcal{S}$ contains both $x_{k-1}^{j}$ and $x_{k}^{j}$ (that is, the stencil crosses the singularity) the error becomes

$$
f(x)=\mathcal{I}_{l}(x)+O([f]),
$$

where $[f]$ stands for the jump of $f$ at the discontinuity. In other words, the accuracy of the prediction on the corresponding interval is reduced to zero. More details can be found in [6].

\section{A new multiresolution representation and asso- ciated thresholding}

In this section we introduce a new nonlinear representation for multiresolution. It is based on a nonlinear modification of the multiresolution details coming from an initial scheme in order to obtain a specific adaptation to the presence of discontinuities.

We first focus our attention on the simplest case: the two-point interpolatory scheme $S_{1}$ that reads:

$$
\left\{\begin{array}{l}
\left(S_{1} f^{j}\right)_{2 k}=f_{k}^{j}, \\
\left(S_{1} f^{j}\right)_{2 k+1}=\frac{f_{k}^{j}+f_{k+1}^{j}}{2} .
\end{array}\right.
$$

\subsection{Adapting the two-point interpolatory multiresolution scheme}

The encoding associated to the two-point interpolatory multiresolution scheme $S_{1}$, is given by

$$
\begin{aligned}
f_{k}^{j} & =f_{2 k}^{j+1}, \\
d l i n_{k}^{j} & =f_{2 k+1}^{j+1}-\frac{f_{k}^{j}+f_{k+1}^{j}}{2},
\end{aligned}
$$

\footnotetext{
${ }^{1}$ The divided differences are defined by induction $f\left[x_{k-1}^{j}, x_{k}^{j}\right]:=\frac{f\left(x_{k}^{j}\right)-f\left(x_{k-1}^{j}\right)}{x_{k}^{j}-x_{k-1}^{j}}$ and $f\left[x_{k-m}^{j}, \ldots, x_{k-m+n}^{j}\right]:=\frac{f\left[x_{k-m+1}^{j}, \ldots, x_{k-m+n}^{j}\right]-f\left[x_{k-m}^{j}, \ldots, x_{k-m+n-1}^{j}\right]}{x_{k-m+n}^{j}-x_{k-m}^{j}}$
} 
and the decoding by

$$
\begin{aligned}
f_{2 k}^{j+1} & =f_{k}^{j}, \\
f_{2 k+1}^{j+1} & =\frac{f_{k}^{j}+f_{k+1}^{j}}{2}+d l i n_{k}^{j} .
\end{aligned}
$$

As we have mentioned in the previous section, in smooth regions, using the properties of Lagrange's interpolation, the details verify

$$
d \operatorname{lin}_{k}^{j}=O\left(h_{j}^{2}\right) .
$$

However, in presence of discontinuities, the accuracy is lost and the details verify

$$
\left|\operatorname{dlin}_{k}^{j}\right|=\frac{1}{2}\left|\Delta_{k}^{j}\right|+O\left(h_{j}\right) .
$$

Our aim is to reduce the size of the details in presence of discontinuities. We propose to consider the projection of the detail on the normal to the line $\left[\left(f_{k}^{j}, x_{k}^{j}\right),\left(f_{k+1}^{j}, x_{k+1}^{j}\right)\right]$, see Figure 1 .

After basic algebraic manipulation we obtain that the normal projection of the detail $d l i n_{k}^{j}$ is:

$$
d_{k}^{j}=\frac{d l i n_{k}^{j}}{2^{j} \sqrt{4^{-j}+\left(\Delta_{k}^{j}\right)^{2}}} .
$$

Pythagorean theorem gives $\left|d_{k}^{j}\right| \leq\left|d l i n_{k}^{j}\right|$. Moreover, close to isolated discontinuities, $d_{k}^{j}=O\left(2^{-j}\right)$. The nonlinear multiresolution decomposition that we then propose reads

$$
\begin{aligned}
& f_{k}^{j}=f_{2 k}^{j+1} \\
& d_{k}^{j}=\frac{\left(f_{2 k+1}^{j+1}-\frac{f_{k}^{j}+f_{k+1}^{j}}{2}\right)}{2^{j} \sqrt{4^{-j}+\left(\Delta_{k}^{j}\right)^{2}}}
\end{aligned}
$$

and the reconstruction

$$
\begin{aligned}
f_{2 k}^{j+1} & =f_{k}^{j} \\
f_{2 k+1}^{j+1} & =\frac{f_{k}^{j}+f_{k+1}^{j}}{2}+d_{k}^{j} \cdot\left\{2^{j} \sqrt{4^{-j}+\left(\Delta_{k}^{j}\right)^{2}}\right\}
\end{aligned}
$$




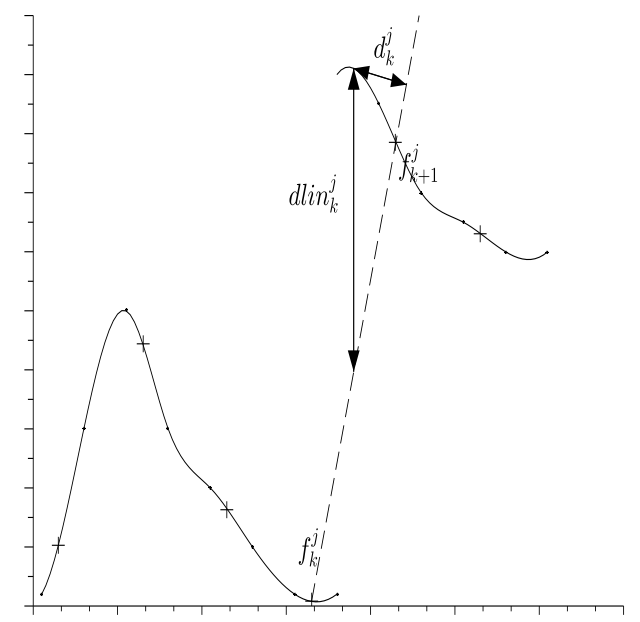

Figure 1: A nonlinear modification of the details using a projection strategy

However, this reduction of the detail size cannot be exploited, without adaption, by a standard tresholding procedure. Indeed, such a thresholding $\tau_{\epsilon}^{j_{0}}$ reads:

Given $j_{0} \leq L-1$ and $\epsilon_{j}>0, j_{0} \leq j \leq L-1$ with $\sum_{j_{0}}^{L-1} \epsilon_{j}=\epsilon$, the thresholding operator $\tau_{\epsilon}^{j_{0}}$ is defined as:

$$
\text { For any } j_{0} \leq j \leq L-1, \text { if }\left|d_{k}^{j}\right| \leq \epsilon_{j} \text { then } \quad \begin{array}{r}
\hat{d_{k}^{j}}=\tau_{\epsilon}^{j_{0}}\left(d_{k}^{j}\right)=0, \\
\text { otherwise } \quad \hat{d_{k}^{j}}=\tau_{\epsilon}^{j_{0}}\left(d_{k}^{j}\right)=d_{k}^{j} .
\end{array}
$$

We propose the following thresholding procedure (see Figure 2)that involves both $d_{k}^{j}$ and $d l i n_{k}^{j}$ (that can be recovered from $f^{j}$ and $d_{k}^{j}$ ):

For any $j_{0} \leq j \leq L-1$ :

$$
\text { If }\left|\operatorname{dlin}_{k}^{j}\right| \leq \epsilon_{j} \text { then } \quad \hat{d}_{k}^{j}=\tau_{\epsilon}^{j_{0}}\left(d_{k}^{j}\right)=0 .
$$




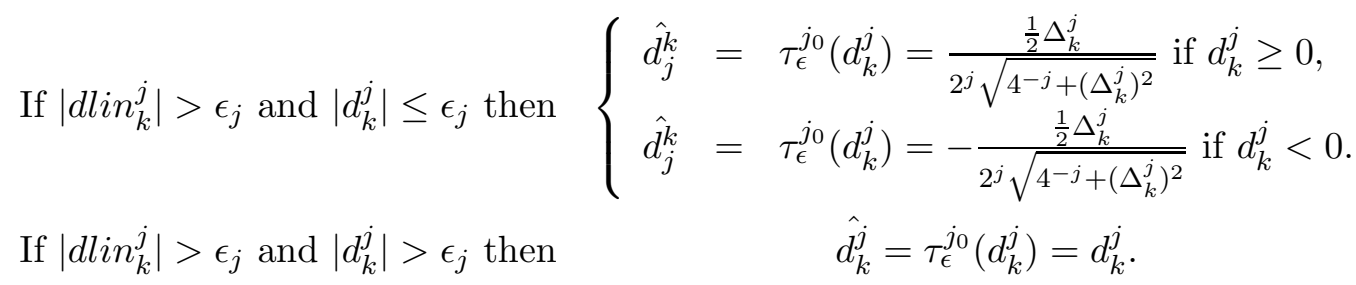

Note that when $\left|\operatorname{dlin}_{k}^{j}\right|>\epsilon_{j}$ and $\left|d_{k}^{j}\right| \leq \epsilon_{j}$ it is only required to store the sign of $d_{k}^{j}$ and, moreover, $\left|\hat{d}_{j}^{k}\right| \leq O\left(2^{-(j+1)}\right)$.

Remark 1 The above procedure takes into account discontinuities at order 0 since it approximates jumps of a piecewise constant function. Approximation at order 1, compatible with the scheme $S_{1}$ reads (see Figure 3):

For any $j_{0} \leq j \leq L-1$ :

$$
\begin{aligned}
& \text { If } \mid \text { dlin }_{k}^{j} \mid \leq \epsilon_{j} \text { then } \quad \hat{d}_{k}^{j}=\tau_{\epsilon}^{j_{0}}\left(d_{k}^{j}\right)=0 . \\
& \text { If } \mid \text { dlin }_{k}^{j} \mid>\epsilon_{j} \text { and }\left|d_{k}^{j}\right| \leq \epsilon_{j} \text { then }\left\{\begin{aligned}
\hat{d_{j}^{k}} & =\tau_{\epsilon}^{j_{0}}\left(d_{k}^{j}\right)=\frac{\frac{1}{2}\left(\Delta_{k}^{j}-\Delta_{k+1}^{j}\right)}{2^{j} \sqrt{4^{-j}+\left(\Delta_{k}^{j}\right)^{2}}} \text { if } d_{k}^{j} \geq 0, \\
\hat{d_{j}^{k}} & =\tau_{\epsilon}^{j_{0}}\left(d_{k}^{j}\right)=-\frac{\frac{1}{2}\left(\Delta_{k}^{j}-\Delta_{k-1}^{j}\right)}{2^{j} \sqrt{4^{-j}+\left(\Delta_{k}^{j}\right)^{2}}} \text { if } d_{k}^{j}<0 .
\end{aligned}\right. \\
& \text { If }\left|\operatorname{dlin}_{k}^{j}\right|>\epsilon_{j} \text { and }\left|d_{k}^{j}\right|>\epsilon_{j} \text { then } \quad \hat{d}_{k}^{j}=\tau_{\epsilon}^{j_{0}}\left(d_{k}^{j}\right)=d_{k}^{j} \text {. }
\end{aligned}
$$

\section{$4 \quad$ Stability analysis}

At this stage, since $d_{k}^{j}$ goes to zero in continuity regions $\left(\Delta_{k}^{j}\right.$ and $d l i n_{k}^{j}$ go to zero at least as $2^{-j}$ ) as well as close to discontinuities, it is clear that the proposed thresholding reduces significantly the number of non zero details. A crucial point is to establish stability, i.e., that the sequence reconstructed from the threshold details lives at a distance from the original sequence controlled by the threshold value.

Considering $\left\{f_{k}^{L}\right\}_{k \in \mathbb{Z}}, j_{0} \leq L-1$ and $\epsilon=\sum_{j_{0}}^{L-1} \epsilon_{j}>0$, we call $\left\{\hat{f_{k}^{L}}\right\}_{k \in \mathbb{Z}}$ the sequence obtained after decomposition, $\tau_{\epsilon}^{j_{0}}$-thresholding and reconstruction, starting from the sequence $\left\{f_{k}^{L}\right\}_{k \in \mathbb{Z}}$. We then have the following result:

Theorem 1 If the sequence $\left\{f_{k}^{L}\right\}_{k \in \mathbb{Z}}$ comes from the sampling of a smooth function but on a finite number of isolated discontinuity points, then, there exists a scale $J_{0}$ such that for any thresholding $\tau_{\epsilon}^{j_{0}}, J_{0} \leq j_{0}$, with $\epsilon_{j}>$ 


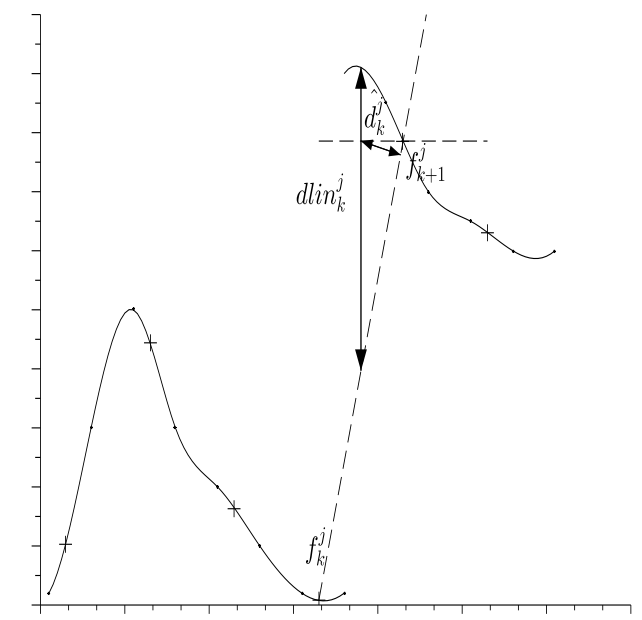

Figure 2: Nonlinear truncation order 0

$O\left(2^{-(j+1)}\right)$

$$
\left\|f^{L}-\hat{f}^{L}\right\|_{\infty} \leq \sum_{j=j_{0}}^{L} \epsilon_{j}:=\epsilon .
$$

Proof:

The proof is detailed for the order 0 procedure but can be adapted to order 1.

We analyze $\left|f_{2 k+1}^{j+1}-\hat{f}_{2 k+1}^{j+1}\right|$ since

$$
\left|f_{2 k}^{j+1}-\hat{f}_{2 k}^{j+1}\right|=\left|f_{k}^{j}-\hat{f}_{k}^{j}\right| .
$$

Two cases have to be considered:

$$
\begin{aligned}
& \hat{f}_{2 k+1}^{j+1}=\frac{\hat{f}_{k}^{j}+\hat{f}_{k+1}^{j}}{2}, \text { or } \\
& \hat{f}_{2 k+1}^{j+1}=\frac{\hat{f}_{k}^{j}+\hat{f}_{k+1}^{j}}{2}+2^{j}\left(\left(\hat{\Delta}_{k}^{j}\right)^{2}+4^{-j}\right)^{1 / 2} \hat{d}_{k}^{j}, \text { with } \begin{cases}\left|d l i n_{k}^{j}\right| & >\epsilon_{j}, \\
\left|\hat{d}_{k}^{j}\right| & <\epsilon_{j} .\end{cases}
\end{aligned}
$$




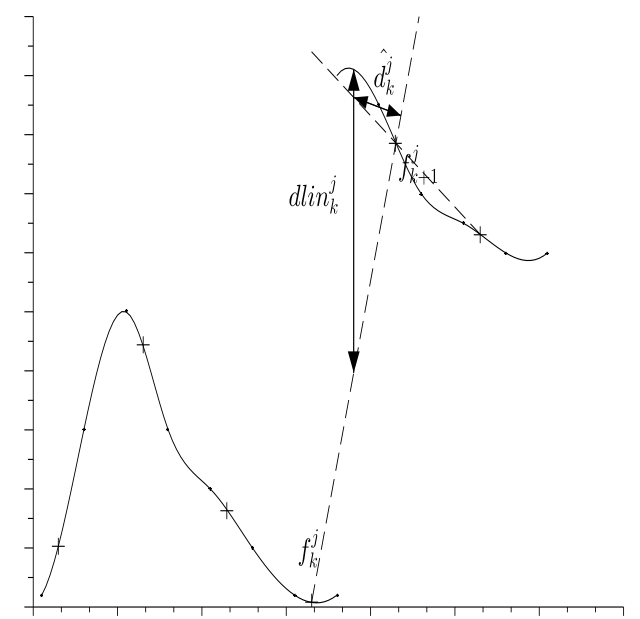

Figure 3: Nonlinear truncation order 1

The first case coincides with the linear case where the stability bounds are known [4]. We then focus our analysis on the second case.

We write

$$
\begin{aligned}
\left|f_{2 k+1}^{j+1}-\hat{f}_{2 k+1}^{j+1}\right|= & \mid\left(\frac{f_{k}^{j}+f_{k+1}^{j}}{2}-\frac{\hat{f}_{k}^{j}+\hat{f}_{k+1}^{j}}{2}\right) \\
& +\left(\left(2^{j}\left(\left(\Delta_{k}^{j}\right)^{2}+4^{-j}\right)^{1 / 2} d_{k}^{j}\right)-\left(2^{j}\left(\left(\hat{\Delta}_{k}^{j}\right)^{2}+4^{-j}\right)^{1 / 2} \hat{d}_{k}^{j}\right)\right) \mid \\
\leq & \mid\left(\frac{f_{k}^{j}+f_{k+1}^{j}}{2}-\frac{\hat{f}_{k}^{j}+\hat{f}_{k+1}^{j}}{2}\right) \\
& +\left(\left(2^{j}\left(\left(\Delta_{k}^{j}\right)^{2}+4^{-j}\right)^{1 / 2} \hat{d}_{k}^{j}\right)-\left(2^{j}\left(\left(\hat{\Delta}_{k}^{j}\right)^{2}+4^{-j}\right)^{1 / 2} \hat{d}_{k}^{j}\right)\right) \mid \\
& +\left|\left(\left(2^{j}\left(\left(\Delta_{k}^{j}\right)^{2}+4^{-j}\right)^{1 / 2} d_{k}^{j}\right)-\left(2^{j}\left(\left(\Delta_{k}^{j}\right)^{2}+4^{-j}\right)^{1 / 2} \hat{d}_{k}^{j}\right)\right)\right|,
\end{aligned}
$$

and analyze the different right hand side terms.

- Bound for the first two terms

The function $F(x):=2^{j}\left(x^{2}+4^{-j}\right)^{1 / 2}$ verifies

$$
\left|\frac{d F(x)}{d x}\right|=\left|2^{j} \frac{x}{\sqrt{x^{2}+4^{-j}}}\right| \leq 2^{j},
$$


then

$$
\begin{aligned}
\left|\left(2^{j}\left(\left(\Delta_{k}^{j}\right)^{2}+4^{-j}\right)^{1 / 2} \hat{d}_{k}^{j}\right)-\left(2^{j}\left(\left(\bar{\Delta}_{k}^{j}\right)^{2}+4^{-j}\right)^{1 / 2} \hat{d}_{k}^{j}\right)\right| & \leq C\left|\Delta_{k}^{j}-\hat{\Delta}_{k}^{j}\right| \\
& \leq C\left|\left(f_{k+1}^{j}-f_{k}^{j}\right)-\left(\hat{f}_{k+1}^{j}-\hat{f}_{k}^{j}\right)\right| \\
& \leq C\left|\left(f_{k+1}^{j}-\hat{f}_{k+1}^{j}\right)-\left(f_{k}^{j}-\hat{f}_{k}^{j}\right)\right|
\end{aligned}
$$

where $C \in \mathbb{R}^{+}$verifies $|C| \leq 2^{j}\left|\hat{d}_{k}^{j}\right| \leq \frac{1}{2}$.

Since $C \leq 1 / 2$ it follows that the first and second terms are bounded by

$$
\left(\frac{1}{2}+C\right)\left\|f^{j}-\hat{f}^{j}\right\|_{\infty}+\left(\frac{1}{2}-C\right)\left\|f^{j}-\hat{f}^{j}\right\|_{\infty}=\left\|f^{j}-\hat{f}^{j}\right\|_{\infty} .
$$

- Bound for the third term

Let us assume, without loos of generality, that $d_{k}^{j}>0$, then $\hat{d}_{k}^{j}=$ $\frac{\Delta_{k}^{j}}{2^{j+1} \sqrt{4^{-j}+\left(\Delta_{k}^{j}\right)^{2}}}$ and the third term coincides with $\operatorname{dlin}_{k}^{j}-\frac{\Delta_{k}^{j}}{2}$. If $k$ corresponds to a smooth region then $\operatorname{dlin}_{k}^{j}=O\left(2^{-(j+1)}\right)$ and $\Delta_{k}^{j}=$ $O\left(2^{-(j+1)}\right)$ while in discontinuity regions $d l i n_{k}^{j}-\frac{\Delta_{k}^{j}}{2}=O\left(2^{-(j+1)}\right)$. In both cases the third term is less than $\epsilon_{j}$ from a given scale $J_{0}$.

Combining the three bounds and iterating, we finally get

$$
\left\|f^{L}-\hat{f}^{L}\right\|_{\infty} \leq \sum_{j=J_{0}}^{L} \epsilon_{j}=\epsilon,
$$

that concludes the proof.

\section{Generalization to centered Lagrange interpola- tion of degree $r$}

A generalization of the previous results can be performed for centered Lagrange interpolation scheme. There, $r=2 p+1$ and $s=p-1$. Using fundamental properties of linear subdivision schemes [12] one get that

$$
\left(S_{r} f^{j}\right)_{2 k+1}=\left(S_{1} f^{j}\right)_{2 k+1}+\left(S_{r, 1} \Delta^{j}\right)_{2 k+1},
$$

where $S_{r, 1}$ is a scheme for the differences $\Delta^{j}$. Moreover, if $r$ is odd and therefore the scheme $S_{r}$ centered, the expression of $\left(S_{r, 1} \Delta^{j}\right)_{2 k+1}$ does not 
contains the term $\Delta_{k}^{j}$. Since in smooth regions $\Delta_{l}^{j}=O\left(2^{-j}\right)$. This implies that, for any value $k$ such that $\left[x_{k}^{j}, x_{k+1}^{j}\right]$ contains a single isolated singularity

$$
\left(S_{r} f^{j}\right)_{2 k+1}=\left(S_{1} f^{j}\right)_{2 k+1}+O\left(2^{-j}\right) .
$$

Therefore, for such values of $k$

$$
\left(S_{r} f^{j}\right)_{2 k+1}=\frac{f_{k}^{j}+f_{k+1}^{j}}{2}+O\left(2^{-j}\right)
$$

The implications of (7) are twofold. First the scheme $S_{r}$ behaves, in the singularity interval as a perturbation of the two point scheme $S_{1}$ and therefore the nonlinear detail constructed following (5) is, up to a perturbation, a normal projection. Second, the stability analysis can be derived similarly, transposing $\frac{f_{k}^{j}+f_{k+1}^{j}}{2}$ by $\left(S_{r} f^{j-1}\right)_{2 k+1}$.

\section{Generalization to the nonlinear scheme PPH}

Up to now, we have concentrated our attention to linear schemes that are known to generate large coefficients close to discontinuities. Nonlinear schemes have been specifically designed to circumvent this drawback.

An archetype for a large class of nonlinear schemes is the scheme $S_{P P H}$ that we recall briefly [3].

Introducing the second order differences $D f_{j}^{k}=f_{k+1}^{j}-2 f_{k}^{j}+f_{k-1}^{j}$, the interpolatory $\mathrm{PPH}$ reconstruction is defined as

$$
\left\{\begin{array}{l}
\left(S_{P P H} f^{j}\right)_{2 k}=f_{k}^{j} \\
\left(S_{P P H} f^{j}\right)_{2 k+1}=\frac{f_{k}^{j}+f_{k+1}^{j}}{2}-\frac{1}{8} H\left(D f_{k+1}^{j}, D f_{k}^{j}\right),
\end{array}\right.
$$

where $H$ is given by:

$$
(x, y) \in \mathbb{R}^{2} \mapsto H(x, y):=\frac{x y}{x+y}(\operatorname{sgn}(x y)+1),
$$

where $\operatorname{sgn}(x)=1$ if $x \geq 0$ and $\operatorname{sgn}(x)=-1$ if $x<0$.

The function $H$ plays indeed a key role. In fact, $S_{P P H}$ has been obtained substituting the arithmetic mean by the function $H$ in the expression of the $S_{3}$ centered Lagrange interpolating scheme. Even if the function $H$ satisfies different properties that lead to a competitive scheme, it appears that when $D f_{k+1}^{j} D f_{k}^{j} \leq 0 S_{P P H}$ coincides with the scheme $S_{1}$. Moreover, this situation occurs in the interval crossing a discontinuity. 
A nonlinear modification of $S_{P P H}$ therefore consists, following (5) in substituting $\left(d_{P P H}\right)_{k}^{j}$ by

$$
d_{k}^{j}=\frac{\left(d_{P P H}\right)_{k}^{j}}{2^{j} \sqrt{4^{-j}+\left(\Delta_{k}^{j}\right)^{2}}} .
$$

\section{Stability via an error-control strategy}

Error-control algorithms have been developed in [1]. They aim to construct a truncation procedure that ensures a prescribed accuracy. They are defined as a modification of classical truncation algorithms that allows to keep track of the cumulative error.

They can be adapted to the above defined truncation procedure. As it will be shown, they lead to a stability result for the nonlinear/error control truncation, without any hypotheses on the initial sequence or the threshold.

The modified encoding procedure reads:

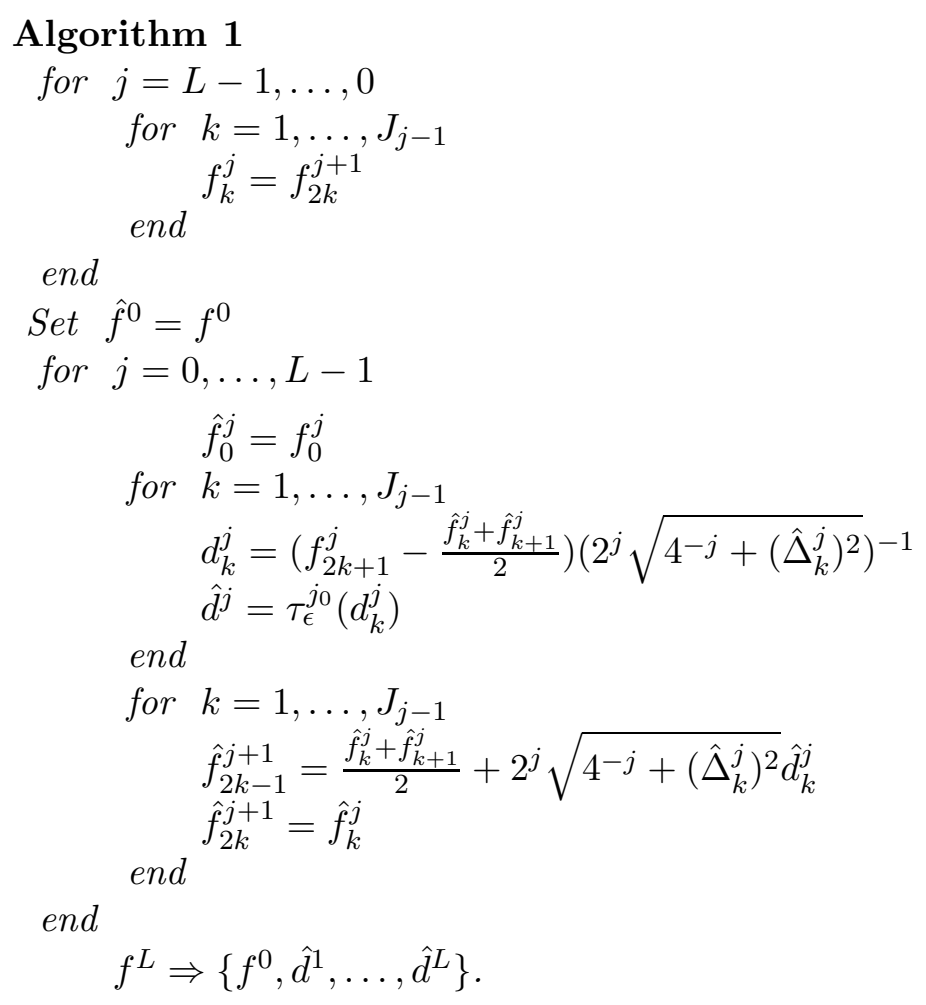


Given a tolerance parameter $T O L$, we consider the following truncation procedure

$$
\hat{d}_{k}^{j}:= \begin{cases}2^{-(j+1)} \operatorname{sgn}\left(d_{k}^{j}\right) & \text { if } 2^{j} \sqrt{4^{-j}+\left(\hat{f}_{k}^{j}-\hat{f}_{k+1}^{j}\right)^{2}}|| d_{k}^{j}\left|-2^{-(j+1)}\right|<T O L, \\ 0 & \text { if } 2^{j} \sqrt{4^{-j}+\left(\hat{f}_{k}^{j}-\hat{f}_{k+1}^{j}\right)^{2}}\left|d_{k}^{j}\right|<T O L, \\ d_{j}^{k} & \text { otherwise. }\end{cases}
$$

Following the numerical results and commentaries of the previous section, taking an adequate parameter $T O L$, a high compression is expected.

Moreover, we have ensured stability properties.

Theorem 2 Given a discrete sequence $f^{L}$ and a tolerance level TOL, then the sequence $\hat{f}^{L}$ satisfies

$$
\left\|f^{L}-\hat{f}^{L}\right\|_{p} \leq T O L
$$

for $p=\infty, 1$ and 2 . Thus, the modified algorithm for the interpolatory case is stable.

Proof:

From the definition of the error-control algorithm we obtain

$$
\begin{aligned}
f_{2 k}^{j+1}-\hat{f}_{2 k}^{j+1} & =f_{k}^{j}-\hat{f}_{k}^{j}, \\
f_{2 k-1}^{j+1}-\hat{f}_{2 k-1}^{j+1} & =f_{2 k-1}^{j+1}-\frac{\hat{f}_{k}^{j}+\hat{f}_{k+1}^{j}}{2}-2^{j} \sqrt{4^{-j}+\left(\hat{\Delta}_{k}^{j}\right)^{2}} \hat{d}_{k}^{j} \\
& =2^{j} \sqrt{4^{-j}+\left(\hat{\Delta}_{k}^{j}\right)^{2}}\left(d_{k}^{j}-\hat{d}_{k}^{j}\right) .
\end{aligned}
$$

Taking norms and using the definition of the truncation procedure the proof is completed.

\section{Numerical experiments}

In this section some numerical experiments are presented. We perform a comparison between the classical implementation and our nonlinear strategy. The evaluated error is the $l^{2}$ error between the initial data and the reconstructed one after compression. We call $n n z$ the number of non zero coefficients after thresholding. 
1. We start with the sampling of a smooth function $f(x)=8 \sin (8 \cdot \pi \cdot x)$, $x \in[0,1]$.

We fix the error after decoding and compute the compression. Both schemes give similar results (see Table 1).

\begin{tabular}{|c|c|c|}
\hline & Nonlinear & Linear (two-points) \\
\hline Error & 0.03 & 0.03 \\
\hline $\mathrm{nnz}$ & 47 & 43 \\
\hline
\end{tabular}

Table 1: Smooth data, $L=8, j_{0}=5$

2. Next, we consider the sampling of the following piecewise smooth function: $f(x)=8 \sin (8 \cdot \pi \cdot x), x \in[0,1 / 2]$ and $f(x)=8 \cos (8 \cdot \pi \cdot x)$, $x \in(1 / 2,1]$.

In this case, we consider a full-compression and compute the error.

Full-compression means for us that

1) For the linear (two-points) scheme we put all the details equal to zero.

2) For our nonlinear multiresolution we put all the details smaller than $2^{-(j+1)}$ equal to zero and the others equal to

$$
\hat{d_{k}^{j}}=\frac{f_{k}^{j}-f_{k+1}^{j}}{2^{j+1}\left(\left(\Delta_{k}^{j}\right)^{2}+4^{-j}\right)^{1 / 2}}\left(\approx \frac{1}{2^{j+1}}\right) .
$$

that is, our data dependent thresholding. Note that in both cases, $n n z=2^{j_{0}}$.

Reconstructed signals are plotted on Figure 4 while errors are gathered in Table 2. Efficiency of the nonlinear approach is quite visible.

\begin{tabular}{|l|c|c|}
\hline & Nonlinear & Linear (Two-points) \\
\hline Error & 0.33 & 6.91 \\
\hline
\end{tabular}

Table 2: Non smooth data, $L=10, j_{0}=7$

\section{Conclusions}

In this paper, a new multiresolution framework has been presented involving a nonlinear evaluation of details and a nonlinear truncation. The corre- 

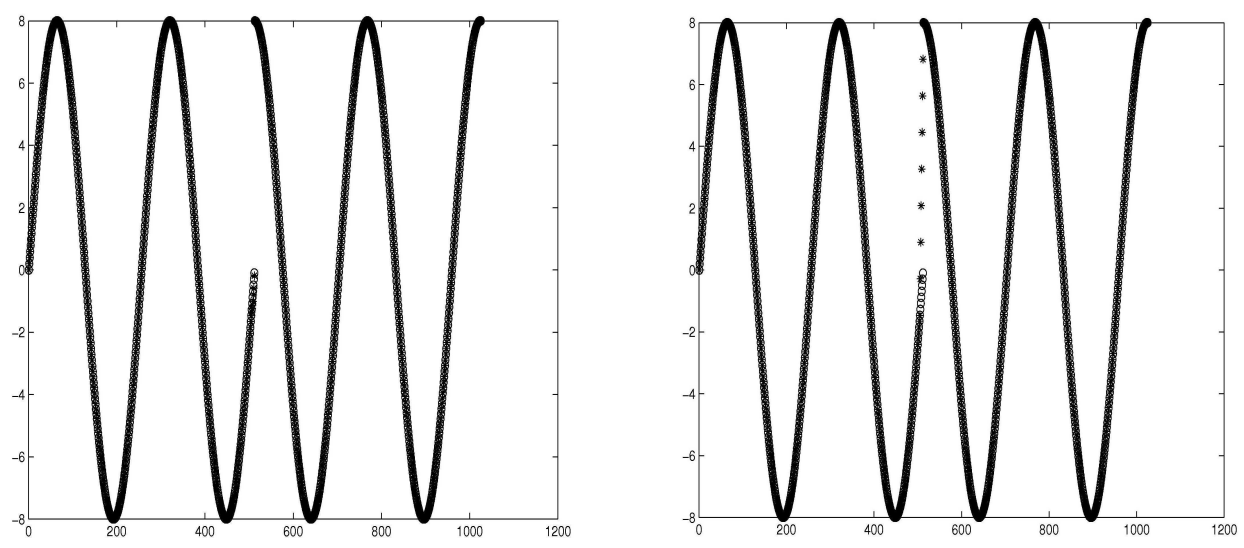

Figure 4: Non linear (left) and linear (right) reconstructions after fullcompression

sponding algorithms have been analyzed in terms of accuracy, compression and stability. It appears that this nonlinear scheme competes favorably with its natural neighboring schemes. 


\section{References}

[1] Amat S., Aràndiga F., Cohen A. and Donat R., (2002). Tensor product multiresolution analysis with error control for compact image representation. Signal Processing, 82(4), 587-608.

[2] Amat S., Aràndiga F., Cohen A., Donat R., Garca G. and von Oehsen M., (2001). Data compression with ENO schemes: A case study. Applied and Computational Harmonic Analysis, 11, 273-288.

[3] Amat S., Donat R., Liandrat J. and Trillo J.C., (2006). Analysis of a new nonlinear subdivision scheme. Applications in image processing. Foundations of Computational Mathematics, 6(2), 193-225.

[4] Amat, S., Donat, R. and Trillo, J. C., (2009). On specific stability bounds for linear multiresolution schemes based on piecewise polynomial Lagrange interpolation. J. Math. Anal. Appl. 358(1), 1827.

[5] Amat S. and Liandrat J., (2005). On the stability of the PPH nonlinear multiresolution, Appl. Comp. Harm. Anal. 18(2), 198-206.

[6] Aràndiga F. and Donat R., (2000). Nonlinear Multi-scale Decomposition: The Approach of A.Harten, Numerical Algorithms, 23, 175-216.

[7] Cohen, A. Theoretical, applied and computational aspects of nonlinear approximation. (English summary) Multiscale problems and methods in numerical simulations, 1-29, Lecture Notes in Math., 1825, Springer, Berlin, 2003.

[8] Cohen A., Dyn N. and Matei B., (2003). Quasilinear subdivision schemes with applications to ENO interpolation. Applied and Computational Harmonic Analysis, 15, 89-116.

[9] Daubechies I., Runborg O. and Sweldens W., (2004). Normal multiresolution approximation of curves, Const. Approx., 20 (3), 399-463.

[10] Delauries G. and Dubuc S., (1989). Symmetric Iterative Interpolation Scheme, Constr. Approx. 5, 49-68.

[11] Donoho D., (1994) Interpolating wavelet transforms. Preprint Standford University.

[12] Dyn N. (1992) Subdivision schemes in computer-aided geometric design. Advances in numerical analysis, II,36-104. 
[13] Guskov I., K. Vidimče K., Sweldens, W. and Schröder P., (2000). Normal meshes. In: Computer Graphics (SIGGRAPH 00 Proceedings). New York: ACM Press, 259268.

[14] Harizanov S., Oswald P. (2010) Stability of nonlinear subdivision and multiscale transforms, Constr. Approx. 31, 359-393.

[15] Harten A., (1996). Multiresolution representation of data II, SIAM J. Numer. Anal., 33(3), 1205-1256.

[16] Matei, B., (2005). Smoothness characterization and stability in nonlinear multiscale framework: theoretical results. Asymptot. Anal., $4 \mathbf{1}$ (3-4), 277-309.

[17] Micchelli C.A., (1996). Interpolatory subdivision schemes and wavelets, Journal of Approximation Theory, 86, 41-71.

[18] Oswald P., (2004). Smoothness of Nonlinear Median-Interpolation Subdivision, Adv. Comput. Math., 20(4), 401-423.

[19] Rabbani M. and Jones P.W., (1991). Digital Image Compression Techniques. Tutorial Text, Society of Photo-Optical Instrumentation Engineers (SPIE), TT07. 